\title{
APLICACIÓN DE UN PLAN \\ DE MANTENIMIENTO CENTRADO \\ EN LA CONFIABILIDAD PARA MEJORAR \\ LA DISPONIBILIDAD DE LA MÁQUINA \\ REMALLADORA DE UNA EMPRESA TEXTIL
}

SOPHIA CRISTINA URIBE*

Universidad de Lima, Perú

Recibido: 28 de febrero del 2020 / Aprobado: 16 de abril del 2020

doi: 10.26439/ing.ind2020.n038.4812

RESUMEN: La presente investigación tuvo como objetivo aplicar un plan de mantenimiento centrado en la confiabilidad de la máquina remalladora de una empresa textil, debido a su baja disponibilidad por paradas prolongadas de mantenimiento correctivo que se presentaban durante la jornada de trabajo. Se elaboró un programa de mantenimiento mediante el análisis de criticidad; luego, se desarrolló el análisis de modo y efecto de fallas para así analizar la situación y escoger, de forma idónea, las acciones a realizar, lo cual permitió mejorar su disponibilidad de manera significativa.

Palabras clave: mantenimiento centrado en la confiabilidad / análisis de modo y efecto de fallas / industria textil

\section{IMPLEMENTATION OF A RELIABILITY-CENTERED \\ MAINTENANCE PLAN TO IMPROVE THE AVAILABILITY \\ OF A TEXTILE COMPANY'S OVERLOCK MACHINE}

ABSTRACT: This research aimed to implement a reliability-centered maintenance plan to improve the availability of a textile company's overlock machine with long corrective maintenance downtime during a workday. A maintenance program was developed using a criticality analysis. Then, a failure mode and effects analysis was conducted to analyze the machine's condition and choose the appropriate actions to be carried out. This process allowed to significantly improve the overlock machine's availability.

Keywords: reliability-centered maintenance / failure mode and effects analysis / textile industry

\footnotetext{
*sophiacabezasuribe@gmail.com
} 


\section{INTRODUCCIÓN}

El mantenimiento centrado en la confiabilidad (RCM, por sus siglas en inglés, reliability centred maintenance) surgió durante la Segunda Guerra Mundial, específicamente, en el sector de la aviación, en donde se registraban alrededor de dos accidentes aéreos diarios, los cuales se originaban por fallas internas del motor del avión. Si bien es cierto, se empezaron a realizar revisiones periódicas que redujeron los accidentes a uno cada dos semanas; aquellas se efectuaban cuando el motor empezaba a fallar (Barreda, 2015).

Hoy en día, el RCM cuenta con un procedimiento para su implementación mediante el cual es pertinente un estudio de los activos fijos, evaluar la criticidad de los equipos, para después, mediante un análisis de fallo, determinar las tareas de mantenimiento, logrando reducir las paradas prolongadas de los equipos y sus costos asociados. Por lo tanto, para la aplicación de un plan de mantenimiento centrado en la confiabilidad es necesario realizar un análisis de modos y efectos de falla (AMEF) y el análisis de criticidad.

Por un lado, el AMEF es un procedimiento de análisis de fallas a través del cual cada falla de un equipo es analizada, de tal forma que permita determinar los efectos sobre las mismas mediante una clasificación de cada falla y así ayudar a reducir la ocurrencia de las mismas (Valdivieso, 2010). Mientras que el análisis de criticidad es una metodología que establece prioridades de procesos, sistemas y equipos, facilitando así la toma de decisiones, enfocándose en las áreas donde más se requiera, para mejorar la confiabilidad operacional según el contexto actual (Salamanca, Velazco y Acevedo, 2016).

La presente investigación tiene como propósito aplicar un plan de mantenimiento centrado en la confiabilidad para una máquina remalladora de una empresa pequeña del sector textil, logrando así prolongar su vida útil, reduciendo el tiempo y los costos que conllevan.

\section{PROBLEMÁTICA}

En la actualidad, la industria textil es considerada como uno de los sectores manufactureros con mayor importancia en la economía peruana, por ello es fundamental que dichas empresas cuenten con un plan de mantenimiento para sus equipos (Ruiz, 2016). Por tanto, al no contar con una matriz de análisis de modos y efectos de fallas de las actividades del mantenimiento, es imposible determinar cuál equipo es el más crítico afectando directamente los costos de producción, ya que se ocasionan paradas prolongadas por falta de repuestos o capacitación del personal, referido al mantenimiento autónomo. Por las razones expuestas, el objetivo de la investigación es elaborar un plan de mantenimiento basado en la metodología RCM.

Es importante mencionar que las demandas actuales de las empresas textiles requieren procesos productivos, seguros y confiables para lograr con ello altos niveles 
de producción a través de la utilización óptima de los recursos con los que cuenta (Córdova, 2018).

Para las medianas y grandes empresas es fundamental aplicar estrategias de mantenimiento que permitan incrementar la vida útil de los equipos, mejorando así su disponibilidad y confiabilidad, lo que conlleva a efectos positivos en la productividad.

La empresa textil, en estudio, se dedica a la producción de prendas de 100 \% algodón y algodón pima, para damas y niñas, con las siguientes máquinas:

a) Máquina recta

b) Máquina recubridora

c) Máquina remalladora

d) Cortadora

Con el paso de los años y al surgir empresas cada vez más competitivas, se hace necesario realizar cambios en el área de mantenimiento, para así contar con procesos más eficientes, que conllevarán a lograr una reducción de costos considerable. Es por ello que el mantenimiento ha ido adquiriendo mayor relevancia, debido a que es un factor fundamental en lo que respecta a la optimización del proceso productivo en una empresa, de esta forma se contribuye a mejorar la confiabilidad de los equipos, ya que se reducen las paradas no previstas y, en ocasiones, prolongadas, minimizando costos asociados al mantenimiento y logrando así mejorar la productividad dentro del proceso de producción de una empresa.

Mediante la aplicación del plan RCM, se logrará contar con un mayor control en relación a las fallas más frecuentes, además, según su ocurrencia, sus costos incurridos, su impacto que genera en la producción, entre otros, al identificar la falla más crítica y elaborar un cronograma de mantenimiento con instructivos que serán de ayuda para la empresa.

A continuación, se presentan las fallas recurrentes en las máquinas remalladoras que ocasionaron baja disponibilidad, obtenidas por medio de su respectiva hoja de control de reparaciones:

- Prenda con manchas de aceite

- Rotura de hilo de aguja

- Falla del motor

- Falla de la correa

- Aguja despuntada 
En la figura 1 se observa el diagrama causa-efecto de las fallas mencionadas en la máquina remalladora:

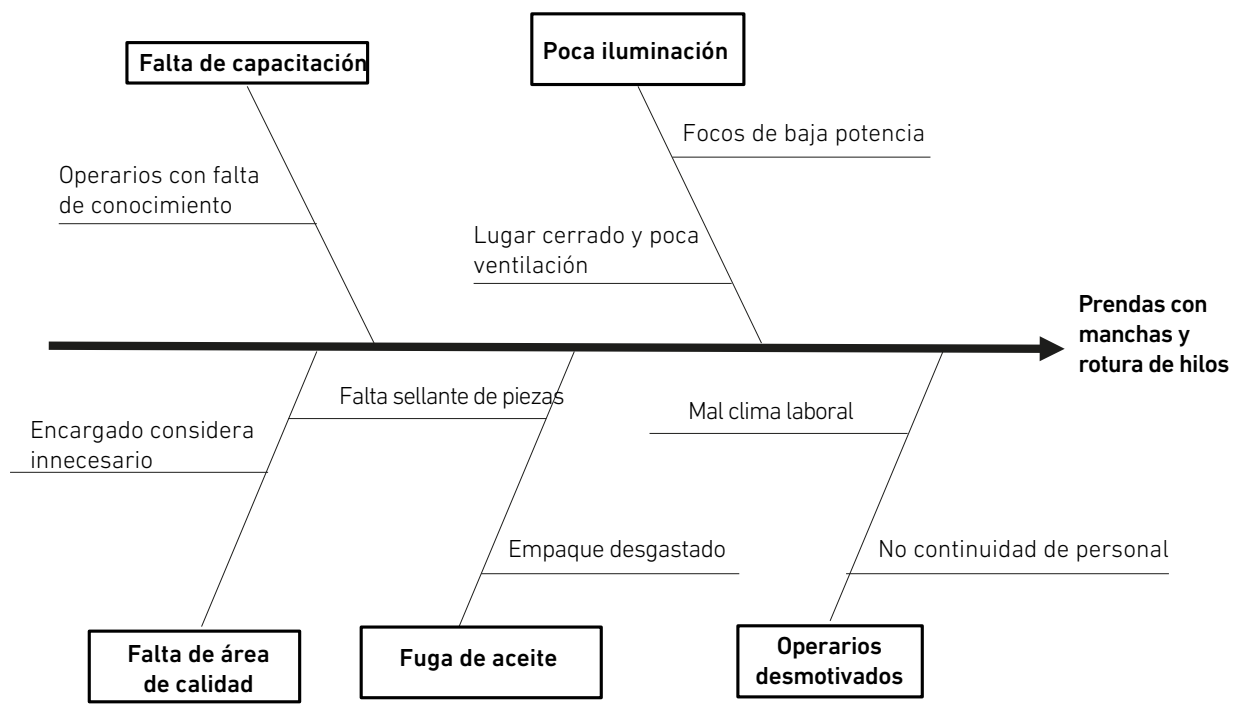

Figura 1. Diagrama causa-efecto de las fallas de prendas con mancha de aceite, rotura del hilo de aguja y aguja despuntada

Elaboración propia

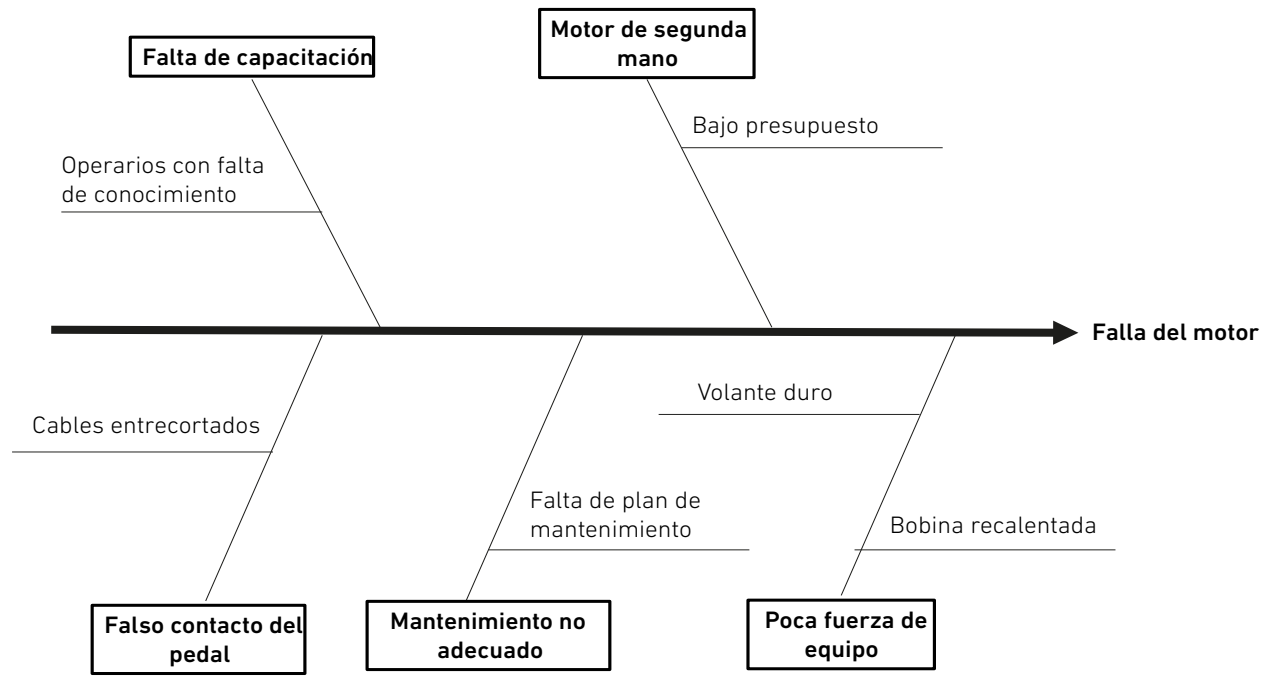

Figura 2. Diagrama causa-efecto de la falla del motor

Elaboración propia 


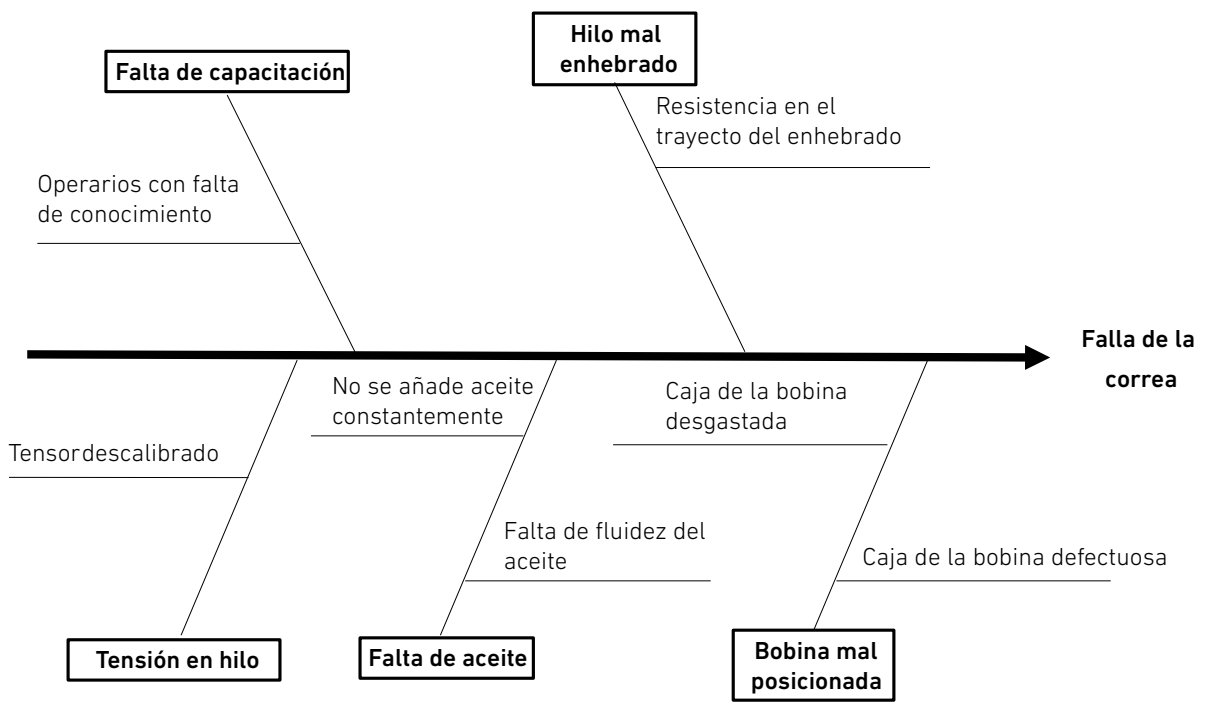

Figura 3. Diagrama causa-efecto de la falla de la correa

Elaboración propia

Las fallas mencionadas, que se presentaban de una manera constante, generaban una serie de mantenimientos correctivos que afectaban directamente a los costos de la empresa, además, al proceso productivo de las prendas de vestir. En la tabla 1 se observa, a modo de resumen, el impacto que estas fallas generaron en la empresa:

Tabla 1

Impacto en la empresa

\begin{tabular}{|c|c|c|c|c|c|}
\hline Descripción & Costo (soles) & $\begin{array}{c}\text { Costo total de } \\
\text { julio a noviem- } \\
\text { bre (soles) }\end{array}$ & $\begin{array}{l}\text { Tiempo pro- } \\
\text { medio por } \\
\text { defecto de julio } \\
\text { a noviembre } \\
\text { (minutos) }\end{array}$ & $\begin{array}{c}\text { Productividad } \\
\text { teórica } \\
\text { (unidades } \\
\text { producidas/ } \\
\text { horas hombre } \\
\text { utilizadas) }\end{array}$ & $\begin{array}{c}\text { Productividad } \\
\text { real (unidades } \\
\text { producidas/ } \\
\text { horas hombre } \\
\text { utilizadas) }\end{array}$ \\
\hline $\begin{array}{l}\text { Prenda con manchas } \\
\text { de aceite }\end{array}$ & 8,50 & 1037,00 & 6773 & 9,1 & 8,95 \\
\hline Rotura de hilo de aguja & 0,0015 & 39,43 & 556 & 9,1 & 9,09 \\
\hline Falla del motor & 150,00 & 1050,00 & 5360 & 9,1 & 8,95 \\
\hline Falla de la correa & 0,0015 & 360,00 & 144 & 9,1 & 9,04 \\
\hline Falla en las cuchillas & 4,00 & 48,00 & 225 & 9,1 & 9,09 \\
\hline Aguja despuntada & 0,50 & 23,14 & 411 & 9,1 & 9,09 \\
\hline Total & 163,00 & 2557,57 & 13469 & & \\
\hline
\end{tabular}

Elaboración propia 
Durante los meses de julio a noviembre del 2019 se obtuvieron las siguientes pérdidas a causa de las fallas mencionadas:

- 122 prendas por manchas de aceite

- 5 prendas por rotura de hilo de aguja

- 124 prendas por falla del motor

- 43 prendas por falla de la correa

- 6 prendas por falla en las cuchillas

- 3 prendas por aguja despuntada

\section{METODOLOGÍA}

El tipo de investigación es aplicada, pues se utilizan los conocimientos para resolver un problema de la empresa. El diseño de investigación es no experimental, ya que no se manipulan las variables (Hernández, Fernández y Baptista, 2014). Asimismo, es transaccional, puesto que la recolección de la información se realizó en un único período de tiempo, de julio a diciembre del 2019.

Los instrumentos utilizados para la investigación fueron los siguientes: para la recolección de la información fue pertinente utilizar la hoja de control de reparaciones, la cual incluye las reparaciones que se realizaron, los costos asociados, los tiempos de operación, entre otros.

Por otro lado, para la aplicación del plan de mantenimiento centrado en la confiabilidad, el instrumento utilizado fue la hoja check list, en la cual se describen las acciones a tener en consideración: diarias, semanales y mensuales. Además, se tuvieron en cuenta las pautas de trabajo que están relacionadas a las acciones de mantenimiento del check list y que se deben realizar en la máquina según los requerimientos.

Para llevar a cabo la metodología RCM, se optó por el procedimiento que se desarrolla en las siguientes etapas (Alegría, 2018):

a) Jerarquizar la criticidad de las máquinas y escoger el más crítico

b) Determinar los modos de falla

c) Determinar los efectos de falla

d) Determinar las causas de las fallas

e) Valorar las consecuencias de las fallas

f) Elaborar un plan de mantenimiento según el árbol lógico de decisiones 
En la figura 4 se detalla un diagrama de bloques de la metodología RCM:

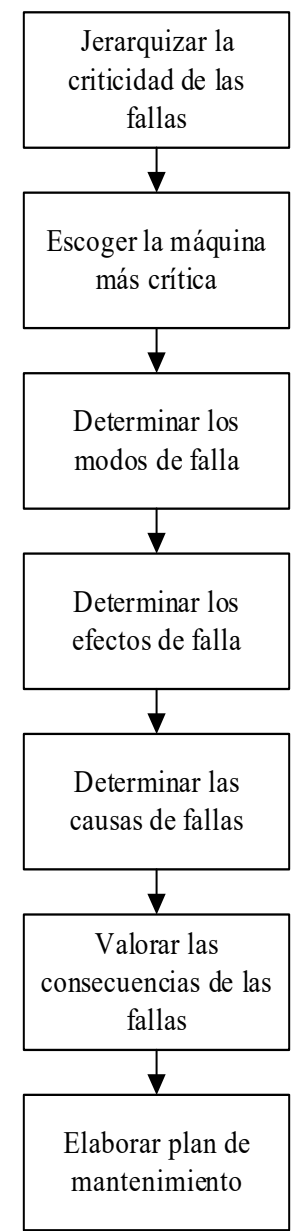

Figura 4. Diagrama de bloques

Fuente: Alegría (2018)

La máquina escogida para la aplicación de la metodología RCM con la herramienta de análisis de modo y efecto de fallas fue la remalladora, ya que era la que presentaba una menor disponibilidad en comparación con las otras máquinas de la empresa, tal como se detalla en la tabla 2. 
Tabla 2

Disponibilidad de las máquinas

\begin{tabular}{lcccc}
\hline \multicolumn{1}{c}{ Descripción } & $\begin{array}{c}\text { Máquina } \\
\text { recubridora }\end{array}$ & $\begin{array}{c}\text { Máquina } \\
\text { recta }\end{array}$ & $\begin{array}{c}\text { Máquina } \\
\text { remalladora }\end{array}$ & Cortadora \\
\hline $\begin{array}{l}\text { Tiempo en reparaciones } \\
\text { en horas }\end{array}$ & 13 & 18 & 45 & 21 \\
$\begin{array}{l}\text { Tiempo planeado en } \\
\text { horas }\end{array}$ & 176 & 176 & 176 & 176 \\
$\begin{array}{l}\text { Porcentaje de } \\
\text { disponibilidad (\%) }\end{array}$ & 92,6 & 89,8 & 74,4 & 88,1 \\
\hline
\end{tabular}

Elaboración propia

Cabe mencionar que para el cálculo de la disponibilidad en cada máquina se utilizó la fórmula (1):

$$
\text { Disponibilidad }=\frac{\text { Tiempo planeado }- \text { Tiempo en reparaciones }}{\text { Tiempo planeado }}
$$

De (1) y de la tabla 2 se obtuvo que la disponibilidad de la máquina remalladora era inferior a la diferencia de las otras máquinas con un valor del 74,4\%.

\section{RESULTADOS}

En primer lugar, se detallaron los tipos de máquinas de la empresa textil, las cuales presentaban fallas con un alto nivel de frecuencia:

Tabla 3

Tipo de máquinas en la empresa textil

\begin{tabular}{lcc}
\hline \multicolumn{1}{c}{ Tipo de máquina } & Modelo & Cantidad \\
\hline Máquina recubridora & Mf-7523 & 3 \\
Máquina recta & Ddl 8100 & 2 \\
Máquina remalladora & Mo-644d & 2 \\
Cortadora & Brute Eastman & 1 \\
\hline
\end{tabular}

Elaboración propia 


\subsection{Análisis de criticidad}

Después de haber recopilado el número de fallas y el impacto económico de estas en la empresa, fue pertinente hacer un análisis de criticidad para identificar las máquinas de mayor riesgo, según su importancia; para ello se realizó un análisis de modos y efectos de fallas para identificar las actividades apropiadas de mantenimiento.

Para jerarquizar los equipos, se procedió a realizar una evaluación del riesgo, que se representa de la siguiente manera (Concha, 2017):

$$
\text { Riesgo }=F F x(I O x F O+C M+S O)
$$

donde:

$F F$ : frecuencia de fallas en un mes de trabajo

IO: impacto operacional que se produce debido a una falla en una determinada máquina

FO: flexibilidad operacional, que está referido al tiempo que podría demorar la reparación de una de las máquinas

CM: costo promedio en reparar una falla de una máquina específica

SO: seguridad ocupacional o instalaciones en caso de ocurrir una falla

Tabla 4

Frecuencia de falla

\begin{tabular}{lc}
\hline Frecuencia de fallas & Cifra \\
\hline Mayor a 7 fallas/mes & 8 \\
Entre 5 y 7 fallas/mes & 6 \\
Entre 2 y 4 fallas $/$ mes & 4 \\
Menor a 2 fallas/mes & 2 \\
\hline
\end{tabular}

Fuente: Concha (2017)

Tabla 5

Impacto operacional de la falla

\begin{tabular}{lc}
\hline \multicolumn{1}{c}{ Impacto operacional } & Cifra \\
\hline Detiene sistema productivo & 12 \\
Disminuye producción & 8 \\
Produce costos operacionales & 5 \\
No genera un efecto negativo & 1 \\
\hline
\end{tabular}

Fuente: Concha (2017) 
Tabla 6

Flexibilidad operacional de la falla

\begin{tabular}{lc}
\hline \multicolumn{1}{c}{ Flexibilidad operacional } & Cifra \\
\hline No existe repuesto en stock & 6 \\
Repuestos compartidos & 3 \\
Repuesto disponible en stock & 1 \\
\hline
\end{tabular}

Fuente: Concha (2017)

Tabla 7

Costo de mantenimiento

\begin{tabular}{lc}
\hline \multicolumn{1}{c}{$\begin{array}{c}\text { Costos de mantenimiento } \\
\text { en soles }\end{array}$} & Cifra \\
\hline De 151 a más & 10 \\
De 101-150 & 7 \\
De $51-100$ & 4 \\
De $0-50$ & 1 \\
\hline
\end{tabular}

Fuente: Concha (2017)

Se procedió a realizar una evaluación de las máquinas que conforman el proceso de producción de las prendas de vestir, siendo sometido al juicio del gerente general de la empresa. A continuación, se detalla los resultados al aplicar la fórmula (2):

Tabla 8

Jerarquización de las máquinas

\begin{tabular}{lcccccc}
\hline \multicolumn{1}{c}{ Jerarquización } & Frecuencia & I.O. & F.O. & C.M. & S.0. & Total \\
\hline Máquina recubridora & 4 & 5 & 3 & 1 & 1 & 68 \\
Máquina recta & 2 & 1 & 3 & 1 & 1 & 10 \\
Máquina remalladora & 8 & 5 & 3 & 4 & 1 & 160 \\
Cortadora & 2 & 2 & 6 & 4 & 1 & 34 \\
\hline
\end{tabular}

Elaboración propia

De la tabla 8, destaca la máquina remalladora por dos factores fundamentales; en primer lugar, la frecuencia de fallas que presenta cada mes y, en segundo lugar, el impacto operacional que conlleva a mayores costos operacionales. Asimismo, es la que representa un menor índice de disponibilidad tal como se detalló en la tabla 8 . Por lo tanto, fue materia de estudio para el análisis de modos y efectos de fallas. 


\subsection{Análisis de modos y efectos de fallas}

En la tabla 9 se detalla el análisis de modos y efectos de fallas de la máquina remalladora.

Tabla 9

Análisis de modos y efectos de fallas de la máquina remalladora

\begin{tabular}{|c|c|c|c|c|}
\hline & Componentes: & AMEF número: & & 1 \\
\hline & Pie prensa telas & Página: & & 1 \\
\hline & Impelentes & & & \\
\hline & Barra de la aguja & & & \\
\hline & Placa de la aguja & Fecha de inicio: & & $1 / 11 / 2019$ \\
\hline & Tira hilo superior & Fecha de fin: & & $31 / 01 / 2020$ \\
\hline & $\begin{array}{l}\text { Mecanismo } \\
\text { diferencial }\end{array}$ & & & \\
\hline & Guardas de aguja & & Responsables: & \\
\hline Máquina remalladora & Garfio & & efe de producció & \\
\hline & $\begin{array}{l}\text { Regulador de } \\
\text { puntada }\end{array}$ & & Analista de calida & \\
\hline & Conjunto de tensores & & Técnico mecánic & \\
\hline & $\begin{array}{l}\text { Mecanismos de } \\
\text { cuchillas }\end{array}$ & & & \\
\hline & $\begin{array}{l}\text { Filtro y visor de } \\
\text { aceite }\end{array}$ & & & \\
\hline & Conjunto de volante & & & \\
\hline & Cabezal & & & \\
\hline Funciones & Falla funcional & Modo de falla & $\begin{array}{c}\text { Frecuencia de } \\
\text { ocurrencia de } \\
\text { falla }\end{array}$ & Efectos de falla \\
\hline $\begin{array}{l}\text { 1. Sirve para el acabado del } \\
\text { borde de las prendas. }\end{array}$ & $\begin{array}{l}\text { Prenda con manchas } \\
\text { de aceite }\end{array}$ & $\begin{array}{l}\text { Desprende } \\
\text { aceite }\end{array}$ & 8 & $\begin{array}{l}\text { Rehacer la } \\
\text { prenda }\end{array}$ \\
\hline $\begin{array}{l}\text { 2. Permite remallar y } \\
\text { terminar costuras internas o }\end{array}$ & $\begin{array}{l}\text { Rotura de hilo de } \\
\text { aguja }\end{array}$ & $\begin{array}{l}\text { Salto de } \\
\text { puntada }\end{array}$ & 8 & $\begin{array}{l}\text { Desgaste de la } \\
\text { aguja }\end{array}$ \\
\hline colocar complementos. & Falla del motor & Puntada floja & 4 & $\begin{array}{l}\text { Se detiene la } \\
\text { producción }\end{array}$ \\
\hline & Falla de la correa & $\begin{array}{l}\text { Mucho ruido al } \\
\text { funcionar }\end{array}$ & 4 & $\begin{array}{l}\text { Reducción de la } \\
\text { eficiencia en el } \\
\text { proceso }\end{array}$ \\
\hline & No corta las prendas & $\begin{array}{l}\text { Cuchillas sin } \\
\text { afilar }\end{array}$ & 6 & $\begin{array}{l}\text { Reducción de la } \\
\text { eficiencia en el } \\
\text { proceso }\end{array}$ \\
\hline & Aguja despuntada & Rotura de aguja & 6 & $\begin{array}{l}\text { Reducción de la } \\
\text { eficiencia en el } \\
\text { proceso }\end{array}$ \\
\hline
\end{tabular}

Elaboración propia 


\subsection{Análisis de las consecuencias de las fallas}

Para un mejor análisis de los efectos de cada modo de falla y la evaluación de las consecuencias, se tuvo en consideración un análisis de criticidad para jerarquizar las fallas, permitiendo así una mejor toma de decisiones con respecto a las tareas de mantenimiento. En la fórmula (3) se presenta el número de prioridad de riesgo (NPR):

$$
N P R=S x O x D
$$

donde:

$S$ : severidad, es decir, la estimación de la gravedad del efecto del modo de falla

$O$ : ocurrencia, la probabilidad de que una causa en específico resulte en un modo de falla $D$ : detección, el valor para clasificar la probabilidad de encontrar una falla antes de que se lleve a cabo

Tabla 10

Escala de severidad, ocurrencia y detección

\begin{tabular}{|c|c|c|c|}
\hline Intervalo & Severidad (S) & Ocurrencia (0) & Detección (D) \\
\hline $9-10$ & Muy alta severidad & $\begin{array}{l}\text { Muy alta probabilidad } \\
\text { de ocurrencia }\end{array}$ & Casi imposible de detectar \\
\hline $6-8$ & Inconveniente mayor & $\begin{array}{l}\text { Alta probabilidad de } \\
\text { ocurrencia }\end{array}$ & Baja capacidad de detección \\
\hline $3-5$ & Inconveniente menor & $\begin{array}{l}\text { Moderada probabilidad } \\
\text { de ocurrencia }\end{array}$ & Alta capacidad de detección \\
\hline $1-2$ & Mínimo efecto & $\begin{array}{l}\text { Baja probabilidad } \\
\text { de ocurrencia }\end{array}$ & $\begin{array}{l}\text { Muy alta capacidad de } \\
\text { detección }\end{array}$ \\
\hline
\end{tabular}

Fuente: Concha (2017)

En la tabla 11 se presenta la clasificación de los modos de falla a que fue sometida, a juicio del gerente general de la empresa, de acuerdo al nivel de riesgo según la fórmula (3):

Tabla 11

Nivel de riesgo

\begin{tabular}{lcccc}
\hline Modos de falla & Severidad & Ocurrencia & Detección & Riesgo \\
\hline Desprende aceite & 6 & 4 & 5 & 120 \\
Salto de puntada & 3 & 4 & 3 & 36 \\
Puntada floja & 7 & 5 & 6 & 210 \\
Mucho ruido al funcionar & 3 & 4 & 3 & 36 \\
Cuchillas sin afilar & 4 & 6 & 3 & 120 \\
Rotura de aguja & 3 & 5 & 45 \\
\hline
\end{tabular}

Elaboración propia 
De la tabla 11, se puede apreciar que el modo de falla fue la puntada floja la que representó un mayor impacto, siendo una falla funcional por falla del motor, ya que cuando ocurrió se detuvo la producción, lo que conllevó a una reducción de la eficiencia de la producción.

En la figura 5 se detalla el árbol lógico de decisiones que se tuvo en cuenta para la elaboración del plan de mantenimiento centrado en la confiabilidad.

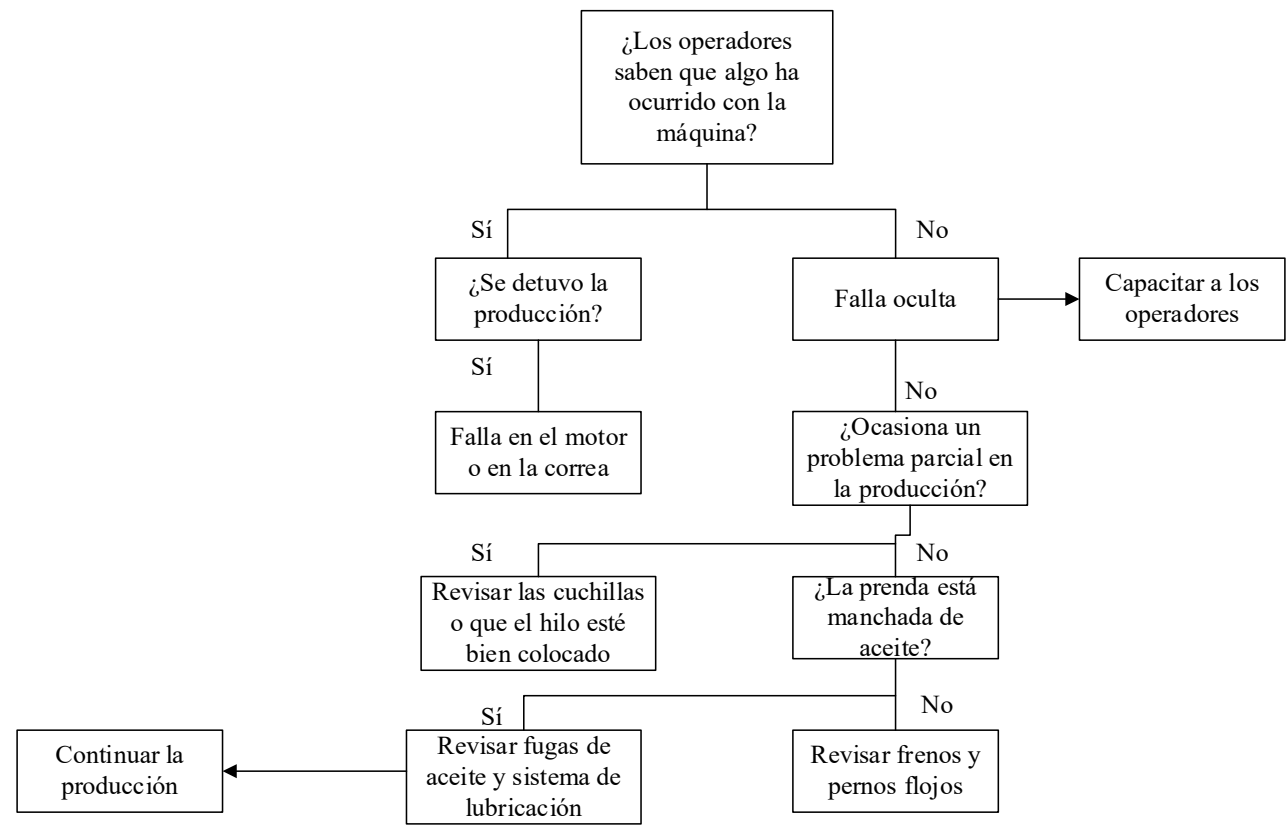

Figura 5. Árbol lógico de decisión RCM

Elaboración propia

De acuerdo con las fallas descritas del análisis de modos y efectos de falla, estas se originaron debido a la aplicación de un mantenimiento correctivo; es decir, cuando la falla ya se había presentado.

Para la aplicación de un plan de mantenimiento centrado en la confiabilidad, se tuvieron en consideración las causas de las fallas, de tal forma que permitieron mejorar la disponibilidad de la máquina en estudio. En la tabla 12 se detalla la frecuencia de mantenimiento de cada actividad. 
Tabla 12

Frecuencia de mantenimiento

\begin{tabular}{lccc}
\hline \multicolumn{4}{c}{ Frecuencia de mantenimiento } \\
\hline & Dectividades & Frecuencia & \\
\cline { 2 - 4 } & $x$ & Semanal & Mensual \\
\hline Limpieza externa & $x$ & & \\
Revisión de sistemas de lubricación & & & \\
Revisión de fugas de aceite & & & $x$ \\
Revisión de pernos flojos & & & $x$ \\
Revisión de sistemas neumáticos & & $x$ & \\
Revisión de ajustes eléctricos & & & \\
Calibración de embragues y frenos & & & \\
Limpieza interna & & & \\
\hline
\end{tabular}

Elaboración propia

En la figura 6 se muestra el cronograma de actividades de acuerdo con la frecuencia de cada actividad, tal como se aprecia, se inició el 6 de enero del 2020 y está programado hasta el 30 de junio, del mismo año, de tal forma que pueda realizarse una segunda revisión de las tareas de mantenimiento que se están ejecutando.

\begin{tabular}{|c|c|c|c|c|c|c|c|c|c|c|c|c|c|c|c|c|c|c|c|c|c|c|c|c|c|c|c|}
\hline \multirow[b]{2}{*}{ Descripción } & \multirow[b]{2}{*}{ Frecuencia } & \multicolumn{4}{|c|}{ Ene-20 } & \multicolumn{4}{|c|}{ Feb-20 } & \multicolumn{5}{|c|}{ Mar-20 } & \multicolumn{4}{|c|}{ Abr-20 } & \multicolumn{5}{|c|}{ May- 20} & \multicolumn{4}{|c|}{ Jun-20 } \\
\hline & & \begin{tabular}{|l|l}
5 & 1 \\
\end{tabular} & 12 & 19 & 26 & 2 & 9 & 16 & 23 & 1 & 8 & 15 & 22 & 29 & 5 & 12 & 19 & 26 & 3 & 10 & 17 & 24 & 31 & 7 & 14 & 21 & 28 \\
\hline $\begin{array}{l}\text { Limpieza } \\
\text { externa }\end{array}$ & Diario & & & & & & & & & & & & & & & & & & & & & & & & & & \\
\hline $\begin{array}{l}\text { Revisión de } \\
\text { sistemas de } \\
\text { lubricación }\end{array}$ & Diario & & & & & & & & & & & & & & & & & & & & & & & & & & \\
\hline $\begin{array}{l}\text { Revisión de } \\
\text { fugas de } \\
\text { aceite }\end{array}$ & Diario & & & & & & & & & & & & & & & & & & & & & & & & & & \\
\hline $\begin{array}{l}\begin{array}{l}\text { Revisión de } \\
\text { pernos flojos }\end{array} \\
\end{array}$ & Semanal & & & & & & & & & & & & & & & & & & & & & & & & & & \\
\hline $\begin{array}{l}\text { Calibración de } \\
\text { embragues y } \\
\text { frenos }\end{array}$ & Semanal & & & & & & & & & & & & & & & & & & & & & & 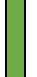 & & & & \\
\hline $\begin{array}{l}\text { Limpieza } \\
\text { interna }\end{array}$ & Semanal & & & & & & & & & & & & & & & & & & & & & & & & & & \\
\hline $\begin{array}{l}\text { Revisión de } \\
\text { sistemas } \\
\text { neumáticos }\end{array}$ & Mensual & & & & & & & & & & & & & & & & & & & & & & & & & & \\
\hline $\begin{array}{l}\text { Revisión de } \\
\text { ajustes } \\
\text { eléctricos }\end{array}$ & Mensual & & & & & & & & & & & & & & & & & & & & & & & & & & \\
\hline
\end{tabular}

Figura 6. Cronograma de mantenimiento

Elaboración propia 
En la tabla 13 se detalla el presupuesto asignado para la ejecución del plan de mantenimiento, cabe mencionar que fue pertinente realizar capacitaciones a los operarios que solían utilizar las máquinas, ya que, de acuerdo con la hoja del control de reparaciones, eran muy repetitivas algunas fallas, como la rotura de hilo de la aguja o las prendas que se manchaban con aceite. Por lo tanto, se está aplicando el mantenimiento autónomo para que detecten, a tiempo, dichas fallas, así como la falla del motor y las otras.

Tabla 13

Presupuesto asignado

\begin{tabular}{lc}
\hline \multicolumn{1}{c}{ Responsable } & Sophia Uribe \\
\hline \multicolumn{1}{c}{ Presupuesto asignado } & Monto (en soles) \\
\hline Supervisión de las actividades de mantenimiento & 1500,00 \\
Repuestos & 500,00 \\
Mantenimiento autónomo & 800,00 \\
Material de apoyo & 200,00 \\
Otros & 100,00 \\
Total & 3100,00 \\
\hline
\end{tabular}

Elaboración propia

Una vez aplicado el plan de mantenimiento, se obtuvo una mejora significativa desde el segundo mes de la puesta en marcha del plan, en relación al indicador de disponibilidad de la máquina remalladora, debido a la reducción en los tiempos de reparación a 14 horas por mes, siendo el tiempo inicial planeado de 176 horas, se procedió a realizar la variación de la disponibilidad en la siguiente ecuación:

$$
\text { Disponibilidad }=\frac{176-14}{176}=92 \%
$$

De acuerdo a (4) se obtuvo un índice de disponibilidad del $92 \%$, incrementando en un 18 \% en relación a la disponibilidad inicial, lo que demostró la importancia de contar con un plan de mantenimiento en una empresa.

\section{CONCLUSIONES}

- De los resultados obtenidos, se destaca la necesidad de prevenir las fallas que pueden ocurrir en las máquinas operadoras, y que interfieren en los tiempos de producción, para mejorar la disponibilidad de las mismas. 
- Se demostró que, con una inversión de 3100 soles, para la puesta en marcha del plan de mantenimiento centrado en la confiabilidad y una capacitación de los operarios para que apliquen el mantenimiento autónomo, se pudo mejorar de forma considerable la disponibilidad de la máquina remalladora.

- Se logró definir el método del mantenimiento centrado en la confiabilidad por medio de equipos de trabajo y delegando acciones a cada trabajador aplicando el método del árbol lógico de decisiones.

- Se diseñó un plan de mantenimiento preventivo como referencia del historial de fallas de las máquinas, análisis de criticidad y el nivel de riesgo.

- Mediante el plan de mantenimiento se aprecia una mejora significativa de la disponibilidad a un $92 \%$ de la máquina remalladora, logrando así el objetivo de la presente investigación.

\section{RECOMENDACIONES}

- Se considera pertinente que la empresa establezca un inventario de repuestos y herramientas como stock de seguridad, de tal forma que garantice una adecuada gestión de mantenimiento.

- Se aconseja realizar un seguimiento de otros indicadores de mantenimiento para evaluar la mejora antes y después de haber aplicado el plan de mantenimiento centrado en la confiabilidad.

- Debe ejecutarse un plan de mantenimiento para las otras máquinas de la empresa para así garantizar la disponibilidad de las mismas y evitar las paradas prolongadas por las reparaciones que se realicen.

- La empresa puede aplicar la metodología del mantenimiento productivo total, ya que los operarios ya se encuentran capacitados con respecto al mantenimiento autónomo para detectar las fallas en las máquinas. Asimismo, se podría alcanzar con dicha metodología una mayor eficiencia de la gestión de mantenimiento.

\section{REFERENCIAS}

Alegría, R. (2018). Aplicación del mantenimiento centrado en la confiabilidad RCM en el sistema de freno neumático de los buses puma katari (tesis para optar el título de mecánico automotriz), Universidad Mayor de San Andrés, La Paz, Bolivia. Recuperada de https://repositorio.umsa.bo/handle/123456789/16748

Barreda, S. (2015). Plan de mantenimiento centrado en la confiabilidad (RCM) en la EDAR de Nules-Vilavella (tesis para optar el título de ingeniero mecánico). Universitat Jaume I, Castellón de la Plana, España. 
Concha, A. A. (2017). Elaboración de plan de mantenimiento frigorífico Fiordosur (tesis para optar el título de técnico en mantenimiento industrial). Universidad Técnica Federico Santa María, Valparaíso, Chile. Recuperada de https://repositorio.usm. $\mathrm{cl} /$ handle/11673/43889

Córdova, K. M. (2018). Implementación de mantenimiento preventivo para la mejora de la productividad en el área de producción de la empresa Creaciones Oswel SAC (tesis para optar el título de ingeniero industrial). Universidad César Vallejo. Recuperada de http://repositorio.ucv.edu.pe/handle/UCV/22921

Diestra, J., Esquivel, L., y Guevara, R. (2017). Programa de mantenimiento centrado en la confiabilidad (RCM) para optimizar la disponibilidad operacional de la máquina con mayor criticidad. Revista Ingeniería: Ciencia, Tecnología e Innovación, 4(1), 1-10.

Espín, H. I. (2018). El RCM (mantenimiento centrado en la confiabilidad) de los equipos del área húmeda y de acabados del cuero de la empresa Tenería Díaz CÍA. LTDA. (tesis para optar el título de ingeniero industrial). Universidad Técnica de Ambato, Ambato, Ecuador. Recuperada de https://repositorio.uta.edu.ec/ handle/123456789/28584

Fornés, R., Ochoa, L., Cano, A., y González, E. (2016). Gestión de mantenimiento centrado en confiabilidad en el área de laboratorios de una institución de educación superior. Revista de Aplicaciones de la Ingeniería, 3(8), 77-86.

Hernández , R., Fernández, C., y Baptista, P. (2004). Metodología de la investigación (5. a ed.). México D. F.: McGraw-Hill.

Ruiz, L. C. (2016). Diseño de un plan de mantenimiento preventivo basado en la confiabilidad para mejorar la disponibilidad y confiabilidad en máquinas circulares en la empresa textil Wg SAC. Innovación en Ingeniería, 2(1), 1-19.

Salamanca, J. E., Velazco, J. A., y Acevedo, A. (2016). Análisis de criticidad y árboles de diagnóstico de fallas para transformadores de potencia. Revista Colombiana de Tecnologías Avanzadas, 1(27), 104-111.

Uzcátegui, J. Y., Varela, A., y Díaz, J. I. (2016). Aplicación de herramientas de clase mundial para la gestión de mantenimiento en empresas cementeras basado en la metodología MCC. Respuestas, 21(1), 77-88.

Valdivieso, J. C. (2010). Diseño de un plan de mantenimiento preventivo para la empresa Extruplas S.A. (tesis para optar el título de ingeniero mecánico). Universidad Politécnica Salesiana, Cuenca, Ecuador. Recuperada de https://dspace.ups.edu. ec/handle/123456789/831 
